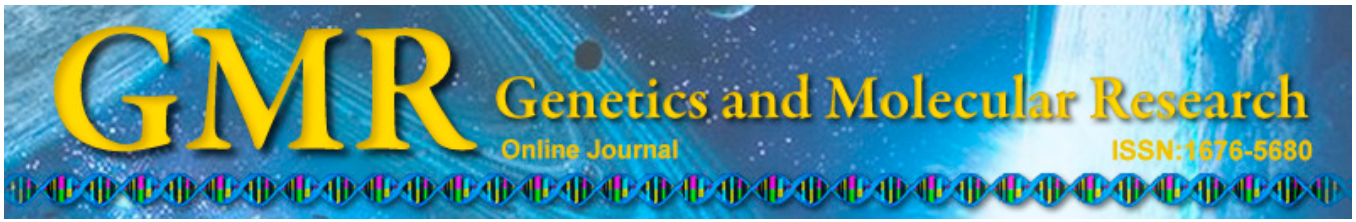

\title{
Characterization of Aspergillus niger endo-1,4- $\beta$-glucanase ENG1 secreted from Saccharomyces cerevisiae using different expression vectors
}

\author{
S.M. Taipakova ${ }^{1}$, I.T. Smekenov ${ }^{1}$, M.K. Saparbaev ${ }^{2}$ and A.K. Bissenbaev ${ }^{1}$ \\ ${ }^{1}$ Institute of Biology and Biotechnology Problems, \\ al-Farabi Kazakh National University, Almaty, Kazakhstan \\ ${ }^{2}$ DNA Repair Group, Institut Gustave Roussy, Villejuif, France \\ Corresponding author: A.K. Bissenbaev \\ E-mail: Amangeldy.Bisenbaev@kaznu.kz
}

Genet. Mol. Res. 14 (2): 6439-6452 (2015)

Received August 28, 2014

Accepted February 9, 2015

Published June 11, 2015

DOI http://dx.doi.org/10.4238/2015.June.11.20

ABSTRACT. Heterologous expression of Aspergillus niger endo-1,4-
$\beta$-glucanase (ENG1) in Saccharomyces cerevisiae was tested both with
an episomal plasmid vector (YEGAp/engl) and a yeast vector capable
of integration into the $H O$ locus of the $S$. cerevisiae chromosome (pHO-
GAPDH-engl-KanMX4-HO). In both cases, engl gene expression in
yeast, with its native signal sequence for secretion, was under the control
of the strong glyceraldehyde 3-phosphate dehydrogenase (GAPDH)
promoter. We aimed to verify how each expression system affects
protein expression, posttranslational modification, and biochemical
properties. Expression of engl from the episomal plasmid vector
YEGAp/engl significantly slowed the growth of a yeast cell culture.
However, expression of engl from the vector integrated into the $H O$
locus of the chromosome did not cause growth suppression, and the
enzyme activity in a culture supernatant was maintained throughout the
incubation time. ENG1 has optimum catalytic activity at pH 6.0 , and 
is stable in the $\mathrm{pH}$ range 5.0-9.0. The enzyme's optimum temperature for catalytic activity at $\mathrm{pH} 6.0$ is $70^{\circ} \mathrm{C}$; importantly, more than $95 \%$ of the enzyme's initial activity remained after a $2-\mathrm{h}$ incubation at $60^{\circ} \mathrm{C}$. The biochemical characterization of ENG1 confirmed the correct expression of the protein and showed that ENG1 expressed by the $\mathrm{pHO}$-GAPDH-engl-KanMX4-HO vector, in addition to its $\mathrm{N}$-linked sites, is overglycosylated at its O-glycosylation sites compared with ENG1 expressed by the YEGAp/engl vector. It is likely that the $\mathrm{O}$-glycosylated form of the $A$. niger ENG1 retains more stable activity during continuous cultivation of recombinant yeasts than the form that is only $\mathrm{N}$-glycosylated.

Key words: Endo-1,4- $\beta$-glucanase; Aspergillus niger; HO locus; Saccharomyces cerevisiae; Episomal plasmid vector; Glycosylation

\section{INTRODUCTION}

Cellulose is the most abundant biological polymer and has a linear structure composed of unbranched D-glucopyranose residues that are joined via 1,4- $\beta$-glucosidic bonds, allowing the molecule to form long, straight chains. Depending on the source, native cellulose can have a very high degree of polymerization: around 10,000 glucose units in wood and 15,000 in cotton (Azizi Samir et al., 2005). Biodegradation of cellulose with the formation of soluble sugars is catalyzed by a specific multi-enzyme cellulase system composed of endo-1,4- $\beta$-glucanase (EC 3.2.1.4), exo-1,4- $\beta$-glucanase (EC 3.2.1.91), exo-1,4- $\beta$-glucosidase (EC 3.2.1.74), and cellobiase (EC 3.2.1.21) (Turon et al., 2008; Horn et al., 2012). Properties of individual enzymes and their interactions in these cellulase complexes determine the efficiency of the hydrolytic degradation of cellulose substrates to soluble oligomeric and monomeric sugars. Glucose, and other sugars produced by the enzymatic hydrolysis of lignocellulosic biomass, can be further converted by certain microorganisms into bioethanol and/or biobutanol.

Among producers of microbial cellulases, the filamentous fungus Aspergillus niger plays a leading role. This fungus secretes a complex set of cellulolytic enzymes, which are utilized in numerous commercial applications such as food, textile, and pharmaceutical industries (De Vries, 2003). A. niger endo-1,4- $\beta$-glucanase, the main component of the cellulases, plays a critical role in the action of the multi-enzyme system. This enzyme is the first to attack the cellulose, hydrolyzing internal $\beta-1,4$-glycosidic bonds at sites remote from the ends of the polymer chain to form cello-oligosaccharides such as mono-, di-, and tri-saccharides (Li et al., 2012).

The yeast episomal plasmid (YEp) has been used to express the A. niger endo-1,4- $\beta$ glucanase gene (engl) in Saccharomyces cerevisiae to study the physical and chemical properties of the enzyme (Hong et al., 2001). However, in the absence of selective pressure, YEp vectors are difficult to maintain stably within cells, and are often lost from the transformed cells during culture growth under non-selective conditions (Yamada et al., 2013). From a practical point of view, yeast-integrated plasmid systems might be more suitable for the stability of the protein, and can also prevent the loss of the recombinant gene in the absence of selective pressure. Recently, $\delta$-integration vectors have been used for stably enhancing target gene copy numbers in S. cerevisiae. A multi-copy gene has been constructed comprising endoglucanase, cellobiohydrolase, and $\beta$-glucosidase genes integrated into the $S$. cerevisiae genome (Yamada 
et al., 2010a,b). This method drastically increased the cellulolytic activity of the strain. However, the integration vectors employed auxotrophic markers such as LEU2-d, HIS3, TRP1, and URA3, and these selective markers differentially influenced the expression of heterologous cellulase genes (Liu et al., 2013). Moreover, these yeast strains might not be suitable for industrial applications, since the industrial yeast strains do not contain internal genetic markers that can identify the need for amino or nucleic acids, for example.

Voth et al. (2001) have reported the establishment of several integral yeast vectors that can be inserted in the yeast chromosome by gene replacement using the homologous sequence portion of the $\mathrm{HO}$ gene. The $H O$ gene encodes an endonuclease responsible for initiating mating type switching and initiation of diploidization of heterothallic yeast (Bakhrat et al., 2004). It was shown that deletion of the $\mathrm{HO}$ locus does not affect the growth of yeast, and that nearly all industrial and laboratory strains of $S$. cerevisiae have a mutation at the $H O$ locus (Baganz et al., 1997; Walker et al., 2003). Moreover, a marker gene for resistance to the antibiotic geneticin (G418), contained in the expression cassette, is dominant and may provide a means for the selection of transformants, regardless of the genotype of the host cell.

In this paper, we present a comparative biochemical analysis of the ENG1 protein expressed using an episomal plasmid vector and a yeast vector that is capable of integration into the $H O$ locus of the $S$. cerevisiae chromosome.

\section{MATERIAL AND METHODS}

\section{Strains and cultivation conditions}

Escherichia coli DH5 $\alpha$ was used for plasmid maintenance and construction. S. cerevisiae strain FF18733 (MATa his7-2 leu2-3,112 lys 1-1 trp1-289 ura3-52) was used as a recipient strain for transformation. E. coli were grown at $37^{\circ} \mathrm{C}$ in Luria-Bertani medium supplemented with ampicillin $(100 \mu \mathrm{g} / \mathrm{mL})$ when necessary. Yeast strains were grown at $30^{\circ} \mathrm{C}$ in YPD medium $[1 \%$ yeast extract $(\mathrm{w} / \mathrm{v}), 2 \%$ peptone $(\mathrm{w} / \mathrm{v}), 2 \%$ glucose $(\mathrm{w} / \mathrm{v})]$. Screening the transformed yeast cells was performed in YPD medium with G418 at a final concentration of $100 \mu \mathrm{g} / \mathrm{mL}$.

\section{Construction of $\mathrm{pHO}$-GAPDH-eng1-KanMX4-HO}

The HO-poly-KanMX4-HO plasmid, kindly provided by David J. Stillman, University of Utah, USA (Voth et al., 2001), carries the KanMX4 selectable marker and two fragments of the $H O$ gene for integration at the $H O$ locus, and was used to construct the yeast expression strain. The cassette containing the A. niger engl gene under the control of the glyceraldehyde 3-phosphate dehydrogenase $(G A P D H)$ promoter and GAPDH terminator was obtained from plasmid YEGAp/engl (Hong et al., 2001), which was kindly provided by Dr. Hidehiko Kumagai, Kyoto University, Japan. To create a vector expressing ENG1, the HindIII fragment from YEGAp/eng1, containing the GAPDH promoter, the A. niger engl gene, and the GAPDH terminator, was blunt-ended and cloned into the blunt and dephosphorylated $\mathrm{SmaI}$ site of the $\mathrm{HO}$-poly-KanMX4-HO plasmid, creating the $\mathrm{HO}$-GAPDH-eng 1 KanMX4-HO expression plasmid vector with flanking homology to target the genes to the $H O$ endonuclease locus (Figure 1). pHO-GAPDH-engl-KanMX4-HO DNA was linearized by treatment with restriction enzymes $X h o \mathrm{I}$ and $\mathrm{XbaI}$ and transformed into yeast cells using a standard lithium acetate method (Gietz and Woods, 2002). YPD medium supplemented 
with filter-sterilized G418 (100 $\mu \mathrm{g} / \mathrm{mL})$ was used for selection and maintenance of the yeast transformants harboring the G418 resistance gene. Yeast transformants displaying ENG1 activity were selected on a $0.5 \%$ carboxymethylcellulose (CMC) YPD medium supplemented with G418 $(100 \mu \mathrm{g} / \mathrm{mL})$ agar plate by the Congo Red halo test. The resulting recombinant strain was designated as FF18733/pHO-GAPDH-eng1-KanMX4-HO. Integration of $\mathrm{pHO}$ GAPDH-engl-KanMX4-HO DNA was confirmed by polymerase chain reaction (PCR) using primers pr1-pr5 (Table 1).

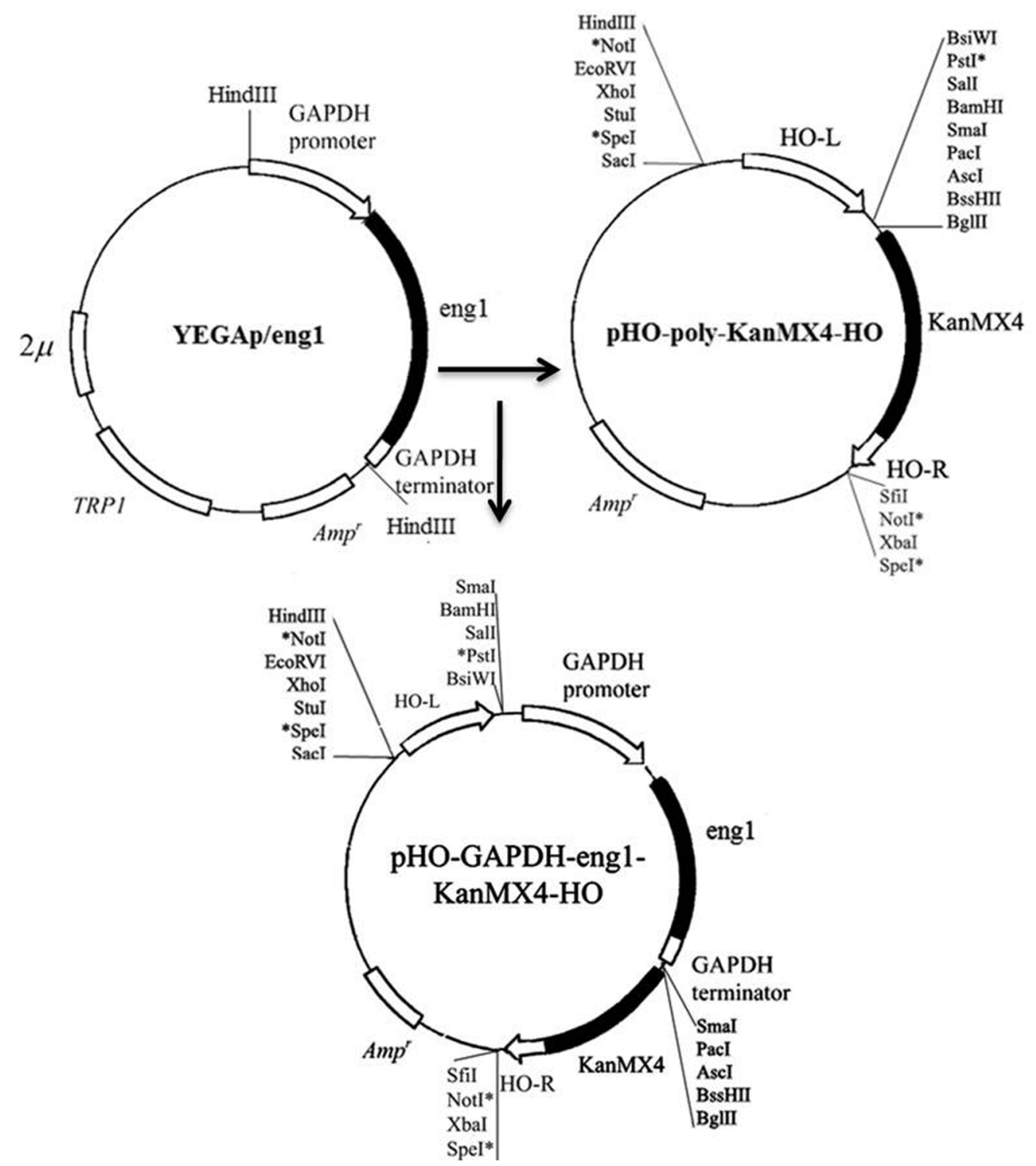

Figure 1. Construction of the integrative vector for the expression of Aspergillus niger endo-1,4- $\beta$-glucanase in yeast. 
Table 1. Oligonucleotide primers used to analyze the efficiency of integration of the integrative vector into the yeast chromosome.

\begin{tabular}{ll}
\hline Primers & DNA sequence (5'-3') \\
\hline Pr-1 & ATTTTAGCAGATGCGCGCACC \\
Pr-2 & GTTTTTCTCAACATTCAAGGAAAG \\
Pr-3 & GGAGGCCCAGAATACCCTCCTTGA \\
Pr-4 & ATGAAGTTTCAGAGCACTCT \\
Pr-5 & TCAAAGATATGCCTCCAGGA \\
\hline
\end{tabular}

Yeast genomic DNA of FF18733/pHO-GAPDH-eng1-KanMX4-HO recombinant strains were isolated according to the method of Harju et al. (2004) and used as a template for amplification by PCR.

Transformation of YEGAp/eng1, a multi-copy shuttle plasmid harboring the TRPI gene, as a selection marker into S. cerevisiae strain FF18733 was carried out by the lithium acetate method. Colonies of transformants showing a phenotype of the prototroph for tryptophan were selected on SD [0.67\% yeast nitrogen base without amino acids (Difco Laboratories, MI, USA), 2\% glucose] agar plates supplemented with appropriate amino acids. Expression of ENG1 was measured by Congo Red staining on culture plates (Teather and Wood, 1982). S. cerevisiae harbored multiple copies of the expression cassette, which was designated as FF18733/YEGAp-engl.

\section{Protein extraction}

Transformed yeast cells were cultured in YPD medium containing 0.1\% CMC, and if necessary supplemented with G418 at a final concentration of $100 \mu \mathrm{g} / \mathrm{mL}$ in a shaker at $30^{\circ} \mathrm{C}$, $200 \mathrm{rpm}$ for 24, 48, 72, 96, and $120 \mathrm{~h}$. Samples were collected at different time points after inoculation, unless specified otherwise. The culture supernatants were obtained by centrifugation ( $5 \mathrm{~min}$ at $3000 \mathrm{~g}$ ) and filtered through a $0.22-\mu \mathrm{m}$ sterile Millipore filter. Protein concentration was determined by the method of Bradford (1976) using bovine serum albumin as the standard. The presence of ENG1 in the culture supernatants of the transformants was verified by sodium dodecyl sulfate-polyacrylamide gel electrophoresis (SDS-PAGE) followed by activity staining. For SDS-PAGE, the culture supernatant was mixed with a saturated solution of ammonium sulfate to $60 \%$ saturation. The suspension was stirred for $12-16 \mathrm{~h}$ and maintained at $4{ }^{\circ} \mathrm{C}$, then centrifuged at $19.500 \mathrm{~g}$ for $30 \mathrm{~min}$. The supernatant was removed and the precipitate was dissolved in $50 \mathrm{mM}$ sodium acetate buffer ( $\mathrm{pH}$ 6.0). Cell growth was monitored by measuring optical density at $600 \mathrm{~nm}$ using a spectrophotometer PD-303UV (Apel Co., Ltd., Japan).

\section{Determination of ENG1 activity}

Enzyme activity was assayed with CMC as a substrate by measuring the amount of reducing sugar according to Miller (1959) with some modifications. The culture supernatant $(1 \mathrm{~mL})$ was pre-warmed at $60^{\circ} \mathrm{C}$ for $5 \mathrm{~min}$ and added to $1 \mathrm{~mL}$ substrate solution $(2 \% \mathrm{CMC}$ in $100 \mathrm{mM}$ sodium acetate buffer, $\mathrm{pH} 6.0$ ), and the mixture was incubated at $60^{\circ} \mathrm{C}$ for exactly 2 $\mathrm{h}$. The reaction was stopped by adding $3 \mathrm{~mL}$ dinitrosalicylic acid solution and boiling for 15 $\mathrm{min}$. The mixture was then cooled immediately and $0.2 \mathrm{~mL}$ reaction solution was diluted with $2.5 \mathrm{~mL}$ distilled water. The amount of reducing sugar was calculated by measuring the absorp- 
tion value at $540 \mathrm{~nm}$. One unit of activity was defined as the amount of enzyme capable of producing $1 \mu \mathrm{M}$ reducing sugar per minute (in $1 \mathrm{mg}$ total protein) under the above conditions.

The optimal $\mathrm{pH}$ for enzyme activity was determined at $60^{\circ} \mathrm{C}$ with $\mathrm{CMC}$. The following buffers used were: sodium acetate, $\mathrm{pH}$ 4.0-6.0; phosphate, $\mathrm{pH} 7.0-8.0$; and $\mathrm{NaOH} / \mathrm{glycine}$, $\mathrm{pH}$ 9.0. The optimal temperature was determined by performing the standard assay at various temperatures from 40 to $80^{\circ} \mathrm{C}$. Thermal stability of the enzyme was estimated by determining residual enzyme activity after incubation at $40^{\circ}-80^{\circ} \mathrm{C}$ for $2 \mathrm{~h}$.

\section{SDS-PAGE and activity staining}

SDS-PAGE was carried out according to the method of Laemmli (1970). SDS-PAGE was performed on gels containing $12 \%$ acrylamide and $0.1 \%$ SDS using a discontinuous Trisglycine buffer system. For detection of ENG1 activity, the proteins were separated on a $12 \%$ polyacrylamide gel with $0.2 \%$ CMC. After SDS-PAGE, the gel was washed twice in a solution containing $50 \mathrm{mM}$ sodium acetate buffer, $\mathrm{pH} 6.0$, and $25 \%$ isopropanol at room temperature to remove the sodium dodecyl sulfate. The proteins were renaturated in a $50 \mathrm{mM}$ sodium acetate buffer, $\mathrm{pH} 6.0$, containing $5 \mathrm{mM} \beta$-mercaptoethanol by rocking the gel for $2 \mathrm{~h}$ at room temperature. The gel was then transferred to a $50 \mathrm{mM}$ sodium acetate buffer, $\mathrm{pH}$ 6.0, and incubated at $70^{\circ} \mathrm{C}$ for another $2 \mathrm{~h}$. The gel was stained with $0.5 \%$ Congo Red for $1 \mathrm{~h}$ and destained in $1 \mathrm{M}$ $\mathrm{NaCl}$. Clear bands on the red background indicated the breakdown of CMC.

\section{Deglycosylation of ENG1}

The ENG1 enzymes were deglycosylated using Endoglycosidase H (Endo H; New England Biolabs, Inc., Ipswich, MA, USA) under denaturing conditions according to manufacturer recommendations. Endo H $(500 \mathrm{U})$ was used to treat $0.02 \mathrm{~mL}$ of concentrated culture medium, estimated at approximately $1 \mathrm{mg} / \mathrm{mL}$ protein. The reactants were incubated overnight at $37^{\circ} \mathrm{C}$ and investigated by SDS-PAGE and enzyme activity staining.

\section{RESULTS}

\section{Transformation and screening of recombinant strains}

To construct a stably expressing yeast strain, we first inserted cDNA of the A. niger ENG1 into the $H O$-poly-KanMX4-HO cassette under the control of the constitutive yeast GAPDH promoter and $G A P D H$ terminator (Figure 1). The resulting expression vector $\mathrm{pHO}-\mathrm{GAPDH}-$ engl-KanMX4-HO (Figure 2A) harboring the gene encoding ENG1 with the native signal peptide for secretion was transformed into S. cerevisiae. Selection of the yeast transformants was performed using YPD agar medium containing the antibiotic G418. In addition, the recombinant clones of S. cerevisiae expressing ENG1 were detected by staining on CMC-supplemented agar plates with $0.5 \%$ Congo Red dye. As shown in Figure 2B, the Congo Red staining of the CMC plates revealed a visible clear zone around the yeast colonies transformed with the $\mathrm{HO}$-GAPDHengl-KanMX4-HO plasmid. Remarkably, no clear zones were visible around the yeast colonies transformed with $H O$-poly-KanMX4-HO plasmid. These data indicate that ENG1 is expressed in an active form and that the native signal peptide attached to the enzyme is recognized by the secretory system of $S$. cerevisiae. Next, we verified the chromosomal integration of the engl 
gene into the $H O$ locus of the yeast chromosome using PCR. For this, the chromosomal DNA from the positive G418-resistant yeast clones was amplified using the primers shown in Table 1, and the reaction products were analyzed by agarose gel electrophoresis. As shown in Figure 2C (lanes 1-2), amplification of the portion of chromosome flanking the $H O$ locus using $\operatorname{Pr} 1$ and $\operatorname{Pr} 2$ primers gave rise to a DNA fragment of the expected size [around 6000 base pairs (bp)], whereas amplification with Pr1 (homologous to the portion of the $H O$ gene) and $\operatorname{Pr} 3$ (homologous to the portion of the KanMX4 gene) primers gave rise to the expected DNA fragment of $2000 \mathrm{bp}$ (Figure 2C, lanes 3-4). Finally, the presence of the A. niger eng1 gene encoding ENG1 in the yeast chromosome was confirmed by amplification of yeast DNA using gene-specific Pr4 and Pr5 primers (Figure 2C, lanes 5-6). Taken together, these results confirm the integration of the cassette $\mathrm{pHO}$-GAPDH-engl-KanMX4-HO into the $\mathrm{HO}$ locus of the genome of S. cerevisiae.
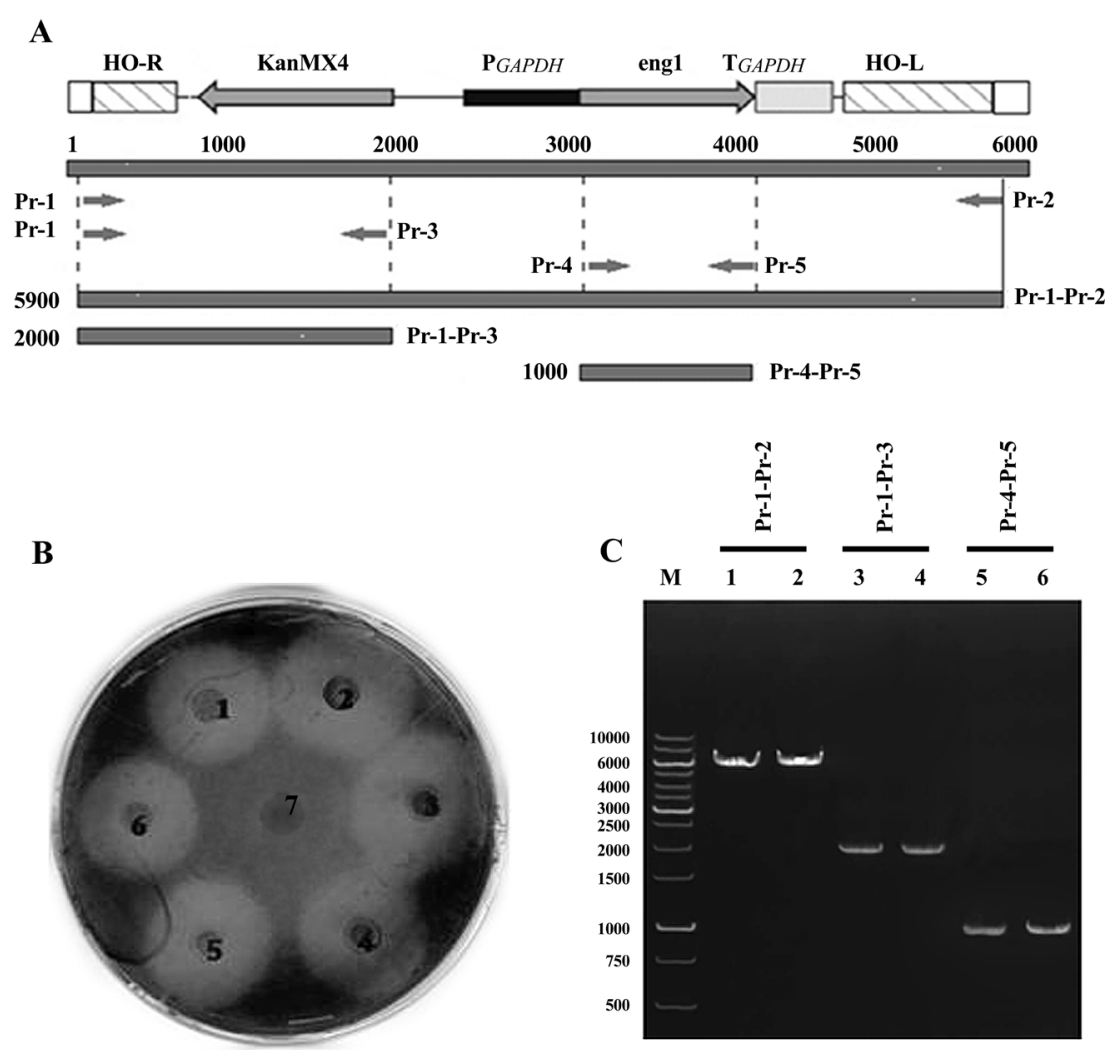

Figure 2. Genomic localization and assay of endo-1,4- $\beta$-glucanase activity on agar plates. A. Integrating vector $\mathrm{pHO}$-GAPDH-eng1-KanMX4-HO contained the Aspergillus niger endo-1,4- $\beta$-glucanase gene fused to the glyceraldehyde 3-phosphate dehydrogenase $(G A P D H)$ promoter. The expression cassette shown was flanked by homology (HO-R and HO-L) regions of the Saccharomyces cerevisiae HO endonuclease gene, which targets integration to the $H O$ locus on chromosome IV. Integration of the $\mathrm{pHO}$-GAPDH-engl-KanMX4-HO cassette was confirmed by polymerase chain reaction (PCR) using primers Pr1-Pr5. The locations and sizes of the PCR products amplified by $\operatorname{Pr} 1-\operatorname{Pr} 5$ are indicated. B. Detection of endo-1,4- $\beta$-glucanase activity in $S$. cerevisiae transformants by cleavage of carboxymethyl cellulose. FF18733/pHO-GAPDH-eng1-KanMX4-HO recombinant yeast cells (16) and untransformed yeast cells (7) were spotted on a carboxymethyl cellulose-supplemented agar plate, and incubated at $30^{\circ} \mathrm{C}$ for 3 days. The plate was then stained with Congo Red. C. Agarose gel electrophoresis of PCR products amplified by $\operatorname{Pr} 1-\operatorname{Pr} 5$ primers. Lane $M=$ GeneRuler 1-kb DNA ladder. 


\section{Expression of the $A$. niger eng1 gene}

The ability of FF18733/pHO-GAPDH-eng1-KanMX4-HO strains to secrete recombinant ENG1 was determined in culture fluids after 24-120 h of incubation (Figure 3).

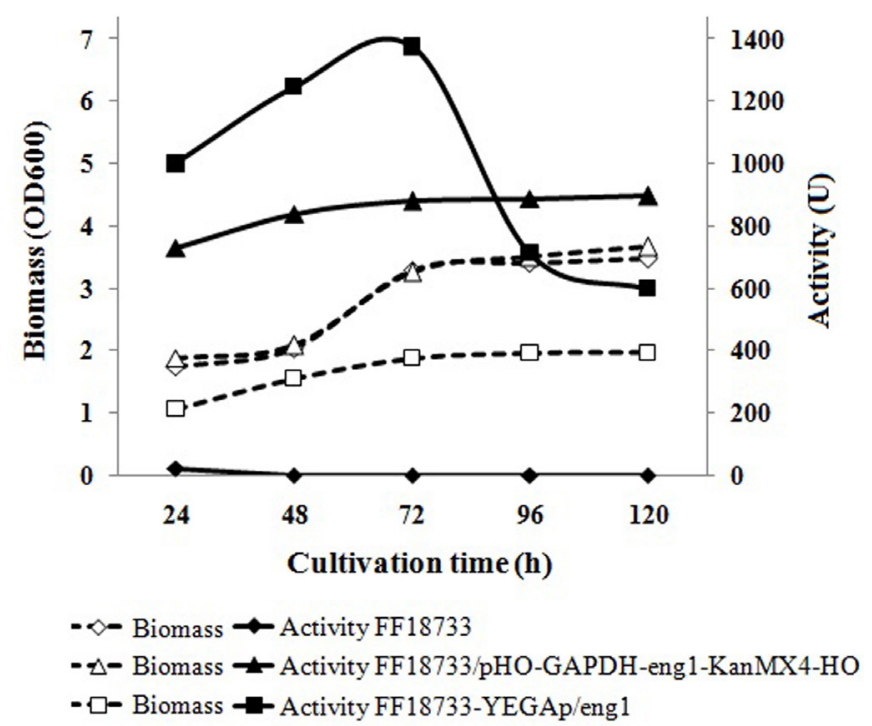

Figure 3. Growth profile of the recombinant Saccharomyces cerevisiae strains and enzyme activity of the secreted endo-1,4- $\beta$-glucanase.

After $48 \mathrm{~h}$ of culture, the activity of the secreted ENG1 rose to 840 units. After $72 \mathrm{~h}$ of culture, the enzyme activity was maintained at 880 units. The culture medium of the control recipient $S$. cerevisiae FF18733 strain showed no ENG1 activity. Importantly, the recombinant strain showed no significant difference in growth rate and biomass yield from the control parent strain, indicating the absence of any negative influence of the heterologous protein production in the yeast FF18733/pHO-GAPDH-engl-KanMX4-HO strain. We also determined the biomass yield and the activity of the secreted recombinant enzyme in the yeast FF18733 strain transformed with the multi-copy shuttle plasmid vector YEGAp/eng1 that contained the gene encoding A. niger ENG1. As shown in Figure 3, the activity of the enzyme secreted by the cultured FF18733/YEGAp-eng1 strain increased significantly within $72 \mathrm{~h}$ of incubation, and was more than 1.5-fold higher than that of the FF18733/pHO-GAPDH-eng1-KanMX4$H O$ strain. A further increase of incubation time of the FF18733/YEGAp-engl culture led to a significant decrease in the activity of the enzyme. Moreover, the FF18733/YEGAp-eng1 strain grew more slowly and gave a lower yield of biomass, as compared with the parental and FF18733/pHO-GAPDH-eng1-KanMX4-HO yeast strains.

Analysis by SDS-PAGE of the cell-free culture broth from the FF18733/pHO-GAPDH-eng1-KanMX4-HO strain revealed a protein band with a molecular weight of approximately $50 \mathrm{kDa}$ (Figure 4A).

Measurement of ENG1 activity in the SDS-PAGE gel using CMC as the substrate showed good agreement between the enzyme activity and the $50-\mathrm{kDa}$ protein, indicating the presence of the secreted A. niger ENG1 in the culture broth (Figure 4B). It should be noted 
that in the yeast cells transformed with the empty control plasmid $H O$-poly-KanMX4- $H O$, no active protein band was detected (data not shown). These data indicate that the major secretory protein in the transformed $S$. cerevisiae cells was the A. niger ENG1.

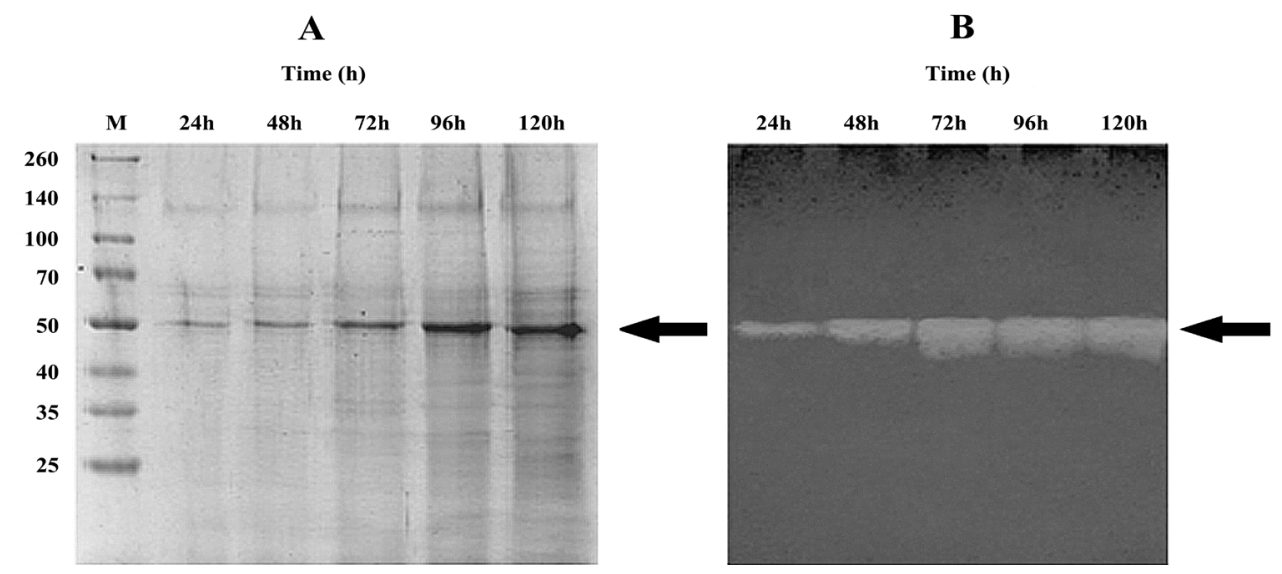

Figure 4. Sodium dodecyl sulfate-polyacrylamide gel electrophoresis (SDS-PAGE) and zymogram of the proteins from the FF18733/pHO-GAPDH-eng1-KanMX4-HO strain culture supernatant. A. Concentrated protein from culture supernatant of FF18733/pHO-GAPDH-eng1-KanMX4-HO on 12\% SDS-PAGE visualized by Coomassie blue staining. Lane $M=$ Spectra Multicolor Broad Range Protein Ladder. B. Zymogram analysis of the SDS-PAGE gel using $0.2 \%$ carboxymethyl cellulose incorporated into the polyacrylamide. Protein samples were taken from the culture after $24-72 \mathrm{~h}$ of incubation at $30^{\circ} \mathrm{C}$.

In subsequent experiments, we studied the secretion of recombinant ENG1 depending on the time of cultivation of FF18733/YEGAp-eng1 yeast strains. Samples for SDS-PAGE and activity staining were obtained every $24 \mathrm{~h}$ from recombinant FF18733/YEGAp-eng1 S. cerevisiae grown in YPD. Interestingly, in contrast to the FF18733/pHO-GAPDH-eng1-KanMX4-HO yeast strains, the multi-copy shuttle plasmid vector YEGAp/engl-harboring yeast strains produced a protein band at $44 \mathrm{kDa}$ (Figure $5 \mathrm{~A}$ ). Moreover, variation in molecular weight and heterogeneity of the ENG1 protein was seen in the activity staining experiment (Figure 5B). The ENG1 from the cultivation of FF18733/YEGAp-eng1 in YPD shifted gradually from a more homogeneous protein in the beginning to a smaller and heterogeneous protein with increasing incubation time. At $72 \mathrm{~h}$, the secreted ENG1 mainly consisted of three active isoforms with molecular weights of 37, 39 , and $44 \mathrm{kDa}$. The proteins with molecular weights of 37 and $39 \mathrm{kDa}$ did not show clear bands in the Coomassie-stained gels, indicating that these proteins were present at low concentrations.

\section{N-glycosylation of the secreted enzyme}

The cDNA of the engl gene of $A$. niger fungus strain IF031125 has been cloned and characterized previously (Hong et al., 2001). The gene encodes a protein consisting of 332 amino acids with a molecular weight of $37 \mathrm{kDa}$. As mentioned above, SDS-PAGE analysis of the ENG1 from the FF18733/YEGAp-eng1 strain revealed the presence of three enzymatically active protein bands with apparent molecular weights of 37, 39, and $44 \mathrm{kDa}$. Interestingly, the molecular weight of the ENG1 secreted by the yeast FF18733/pHO-GAPDH-eng1-KanMX4$H O$ strain based on the SDS-PAGE analysis $(50 \mathrm{kDa})$ was much higher than the calculated 
molecular weight of the enzyme $(37 \mathrm{kDa})$. These observations indicate that the ENG1 protein when expressed in yeast cells has a higher molecular weight than expected. Such an increase in molecular weight can be attributed to protein glycosylation. Glycosylated proteins are often presented in the form of a collection of different molecular weight glycoforms differing in length and composition of the oligosaccharide chains covalently attached to the protein (Gao et al., 2012). To verify this assumption, the amino acid sequence of ENG1 was analyzed for the presence of N-linked glycosylation sites using the National Center for Biotechnology Information database. Based on the analysis of the primary structure of ENG1, we found four potential $\mathrm{N}$-linked and three O-linked glycosylation sites (data not shown).

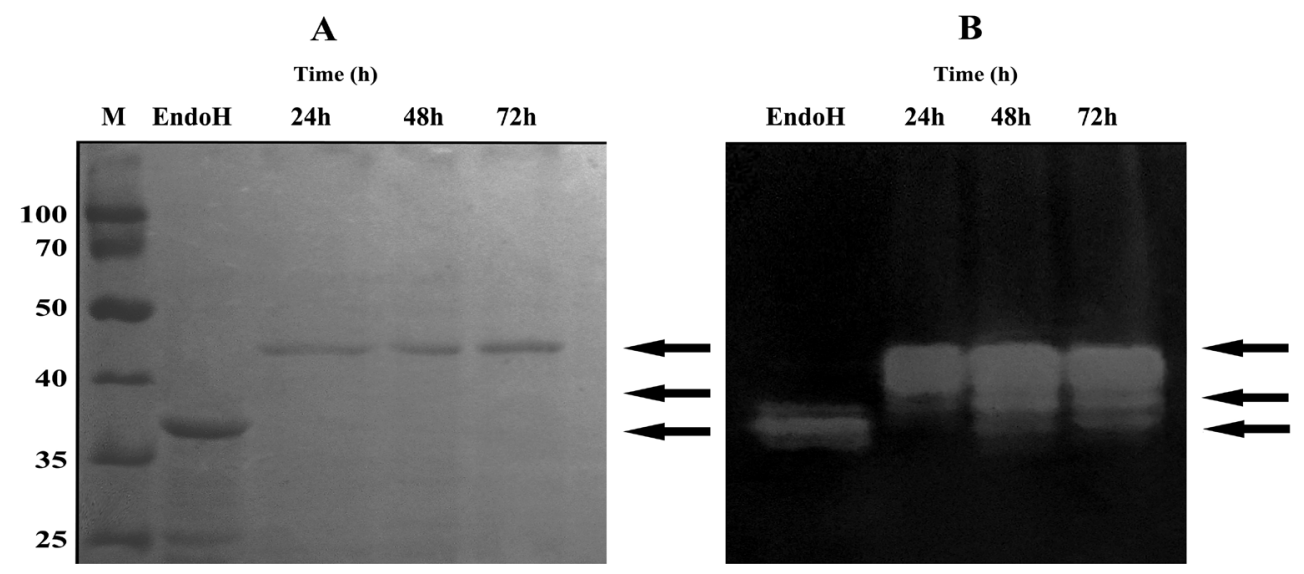

Figure 5. Sodium dodecyl sulfate-polyacrylamide gel electrophoresis (SDS-PAGE) and zymogram of the proteins from the FF18733/YEGAp-eng1 strain culture supernatant treated by Endo $H$. The recombinant endo-1,4- $\beta$ glucanase from the culture supernatant of FF18733/YEGAp-eng1 was deglycosylated by incubation with Endo H protein and then analyzed by SDS-PAGE followed by an enzyme activity test. A. SDS-PAGE. B. Zymogram. Lane Endo $H=$ recombinant endo-1,4- $\beta$-glucanase digested by Endo $\mathrm{H}$; lanes 24-72 $h$ = concentrated protein samples from the culture supernatant of FF18733/YEGAp-eng1 were taken after 24-72 h of incubation; lane $M=$ spectra Multicolor Broad Range Protein Ladder.

The endo- $\beta-\mathrm{N}$-acetylglucosaminidase $\mathrm{H}$ (Endo H, EC 3.2.1.96) is a highly specific endoglycosidase that cleaves asparagine-linked oligosaccharides (N-glycosylation), but not O-linked oligosaccharides (Maley et al., 1989). Therefore, recombinant eng1 from a culture supernatant of FF18733/YEGAp-eng1 was deglycosylated by incubation with Endo H and then analyzed by SDS-PAGE followed by an enzyme activity test. After digestion with Endo $\mathrm{H}$, a single band with a molecular weight corresponding to the expected $37 \mathrm{kDa}$ was observed (Figure 5B). These results indicate that differently glycosylated forms of ENG1 were present in the FF18733/YEGAp-eng1 yeast strain.

Consequently, the extracellular proteins secreted by the FF18733/pHO-GAPDHeng1-KanMX4- $\mathrm{HO}$ strain were incubated with $500 \mathrm{U}$ Endo $\mathrm{H}$ for 2, 6, and $12 \mathrm{~h}$, and then the products of reaction were analyzed by SDS-PAGE. As shown in Figure 6, the Endo H-catalyzed deglycosylation led to a decrease in the molecular weight of recombinant ENG1 until about $45 \mathrm{kDa}$, which is larger than the calculated molecular weight (37 kDa). Furthermore, the enzymatic activity measurements using SDS-PAGE showed similar results, indicating the presence of partially deglycosylated ENG1. 


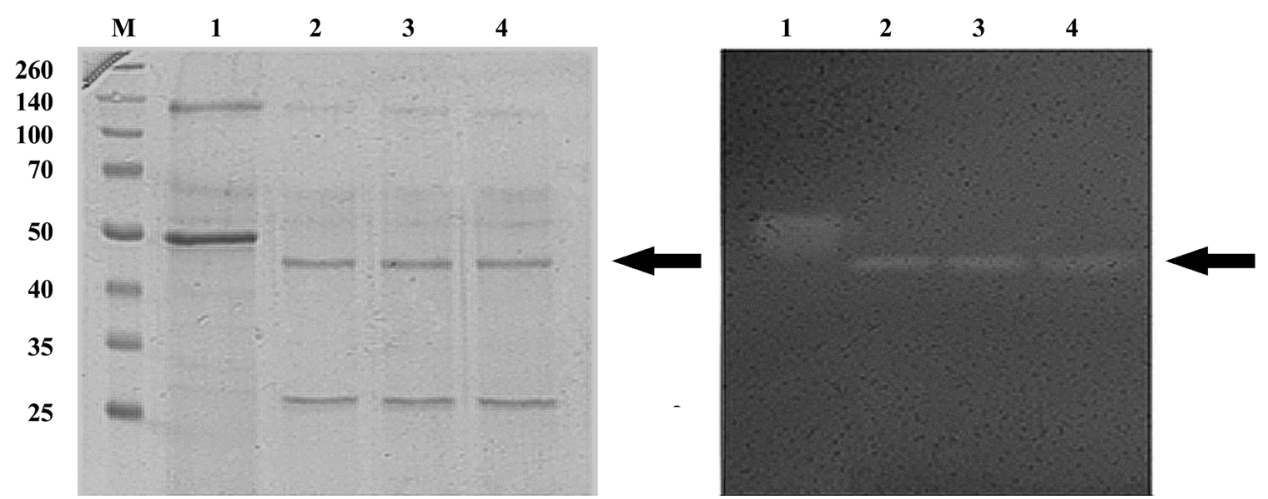

Figure 6. Sodium dodecyl sulfate-polyacrylamide gel electrophoresis and zymogram of the proteins from the FF18733/pHO-GAPDH-engl-KanMX4-HO strain culture supernatant treated by Endo H. Lane 1 = recombinant endo-1,4- $\beta$-glucanase without Endo $\mathrm{H}$ digestion; lanes $2-4$ = endo-1,4- $\beta$-glucanase digested by Endo $\mathrm{H}$; lane $M=$ spectra Multicolor Broad Range Protein Ladder.

\section{Determination of the reaction conditions for the recombinant $A$. niger ENG1}

Characterization of the recombinant enzyme was carried out using CMC as the substrate. To determine the effect of $\mathrm{pH}$ on the stability of the enzyme, the enzyme preparation was incubated in buffers at $60^{\circ} \mathrm{C}$ for $2 \mathrm{~h}$ at various $\mathrm{pH}$ values. The recombinant enzyme demonstrated stability over a broad $\mathrm{pH}$ range, and more than $90 \%$ of total activity remained after incubation in the $\mathrm{pH}$ range of 5.0-9.0 (Figure 7A). Study of the influence of elevated temperature over time on the activity of recombinant ENG1 was performed at $\mathrm{pH}$ 6.0. The recombinant enzyme showed high thermostability; $100 \%$ of total activity remained after incubation of the protein preparation at $60^{\circ} \mathrm{C}$ for $2 \mathrm{~h}$, and $80 \%$ activity remained after incubation at $70^{\circ} \mathrm{C}$ (Figure $7 \mathrm{~B}$ ). Taken together, these results indicate that the recombinant enzyme is very resistant to high temperatures.

A

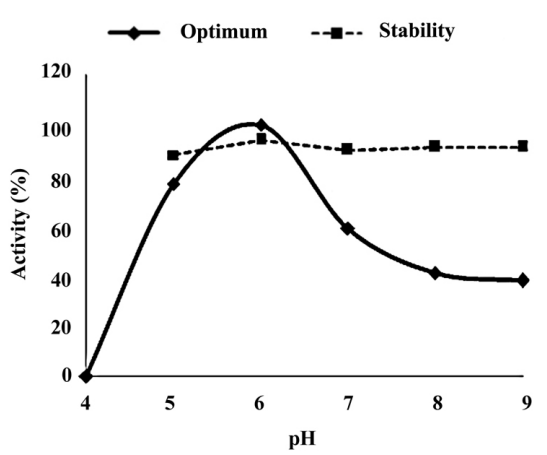

B

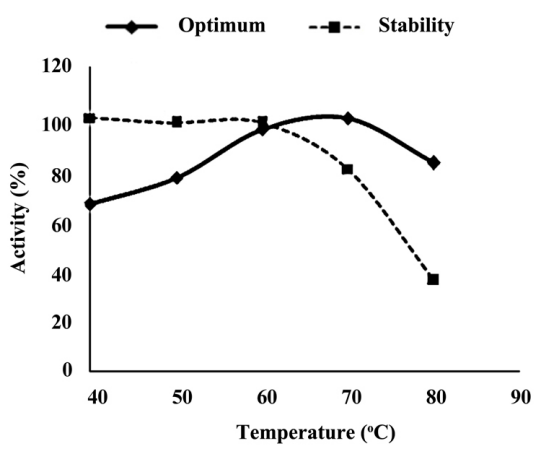

Figure 7. Activity of Aspergillus niger recombinant endo-1,4- $\beta$-glucanase on carboxymethylcellulose as a function of temperature and $\mathrm{pH}$. A. Optimal and $\mathrm{pH}$ stability of endo-1,4- $\beta$-glucanase. To determine $\mathrm{pH}$ stability of the recombinant endo-1,4- $\beta$-glucanase, the protein was incubated at the indicated $\mathrm{pH}$ for $2 \mathrm{~h}$ and the enzyme activity was measured at $60^{\circ} \mathrm{C}$. B. Optimal temperature and temperature stability. To determine temperature stability of the recombinant endo-1,4$\beta$-glucanase, the protein was incubated at the indicated temperatures for $2 \mathrm{~h}$ and the enzyme activity was measured at $70^{\circ} \mathrm{C}$. 


\section{DISCUSSION}

Since A. niger ENG1 has four potential sites for N-glycosylation and three proposed O-glycosylation sites, this enzyme also becomes suitable for a comparison of glycosylation levels when expressed in the integrative and episomal constructs. Moreover, it allows for an evaluation of how different glycosylation levels influence enzyme performance, mainly its activity and other biochemical properties such as stability. For this purpose, we cloned A. niger eng1 cDNA in the episomal plasmid vector YEGAp and a yeast vector capable of integration into the $H O$ locus of the $S$. cerevisiae chromosome. In both cases, engl gene expression in yeast, with its native signal sequence for secretion, was under the control of the strong GAP$D H$ promoter. The $H O$ endonuclease is not expressed in yeast with high ploidy, and is active only in haploid organisms (Hector et al., 2011); deletion of the $H O$ gene does not affect the growth rate and viability of yeast (Baganz et al., 1997; Voth et al., 2001).

When both episomal and integrative expression systems were compared in S. cerevisiae, the activity of ENG1 was higher in the episomal than in the integrative construct at the beginning of cultivation. However, further increase of incubation time led to a significant decrease in the activity of the enzyme and in slower growth of the culture compared to the control strain. In contrast, in the case of the recombinant strain FF18733/pHO-GAPDH-eng1KanMX4-HO, we did not observe suppression of growth, and the ENG1 activity was maintained throughout the incubation time.

Yeast cells transformed with autonomously replicating vector DNAs can contain up to 40 plasmid copies per cell (Ghosh et al., 2007; Jeon et al., 2009). Therefore, continuous expression of a large number of the heterologous proteins in FF18733/YEGAp-eng1 strains might lead to the depletion of energy resources and the disruption of cell metabolism. In addition, the autonomously replicating plasmid is generally difficult to maintain stably in the cells and, as a general rule, a relatively high number of cells lose the plasmid, especially in the absence of selective pressure for the plasmid markers (Yamada et al., 2013).

The biochemical characterization of ENG1 performed here, in terms of molecular weight estimation using denaturing gels, activity at different $\mathrm{pH}$ values, and stability with regards to $\mathrm{pH}$ and temperature, confirms that both yeasts were able to express and secrete the expected heterologous protein.

The $\mathrm{pH}$ for maximal ENG1 activity expressed in the FF18733/pHO-GAPDH-eng1KanMX4- $H O$ strain was 6.0 , and the enzyme was stable over a wide $\mathrm{pH}$ range (5.0-9.0). The optimum temperature at $\mathrm{pH} 6.0$ was $70^{\circ} \mathrm{C}$, and the initial activity of the recombinant ENG1 remained stable during incubation at $60^{\circ} \mathrm{C}$ for $2 \mathrm{~h}$. These data are in agreement with the results reported by Hong et al. (2001), indicating that the stable expression does not alter the properties of the fungal enzyme. A. niger ENG1 secreted by FF18733/pHO-GAPDH-eng1KanMX4- $\mathrm{HO}$ recombinant strains was represented by a single band with a molecular weight of $50 \mathrm{kDa}$. Endo H-catalyzed protein deglycosylation led to a reduction of molecular weight but not to $37 \mathrm{kDa}$. Activity staining of SDS-PAGE revealed at least three active protein bands $(37,39$, and $44 \mathrm{kDa})$ from the culture supernatants of F18733/YEGAp-eng1 strains according to molecular weight. Furthermore, the Endo $\mathrm{H}$-catalyzed protein deglycosylation resulted in the reduction of molecular weight to the expected $37 \mathrm{kDa}$ (Figure $5 \mathrm{~A}$ and $\mathrm{B}$ ), suggesting that a different glycosylated form of the ENG1 enzyme is secreted when it is expressed in episomal constructs. 
It seems that the heterologous ENG1 was more hyperglycosylated in the integrative construct than in the episomal construct, and that hyperglycosylation did not negatively affect enzyme activity or stability. We may speculate that the ENG1 expressed in the FF18733/pHOGAPDH-engl-KanMX4-HO strain is extensively glycosylated at its N-glycosylation sites compared with the enzyme expressed in the FF18733/YEGAp-eng1 yeast strain. To validate our suggestion, the extracellular proteins secreted by the FF18733/pHO-GAPDH-eng1-KanMX4-HO strains were incubated with $500 \mathrm{U}$ Endo $\mathrm{H}$ for 2, 6, and $12 \mathrm{~h}$, and then the products of reaction were analyzed by SDS-PAGE. Interestingly, the prolonged treatment of the glycosylated ENG1 by Endo H did not lead to formation of a completely deglycosylated 37-kDa protein, but only to a relatively small decrease in molecular weight down to $45 \mathrm{kDa}$, estimated from the mobility of the protein in SDS-PAGE. These results indicate that the increased molecular weight of the deglycosylated recombinant $A$. niger ENG1 could be due to additional Olinked glycosylation of the enzyme in the FF18733/pHO-GAPDH-engl-KanMX4- $\mathrm{HO}$ yeast host. Consequently, we propose that the observed differences in the glycosylation pattern of the $A$. niger ENG1 are due to a difference in the posttranslational modifications of the recombinant protein when it is expressed from the chromosomally integrated genes compared to the autonomously replicating and plasmid vectors.

Glycosylation is an important posttranslational modification of proteins that influences their stability, conformation, secretion, and biological activity (Zhang et al., 2012). Olinked glycosylation is important for enzyme stabilization (Chen et al., 2014). It is likely that the O-glycosylated form of ENG1 retains more stable activity during continuous cultivation of recombinant yeasts than the form that is only $\mathrm{N}$-glycosylated.

\section{ACKNOWLEDGMENTS}

Research supported by a grant from the Science Committee of the Ministry of Education and Science of the Republic of Kazakhstan (\#1324/GF04).

\section{REFERENCES}

Azizi Samir MA, Alloin F and Dufresne A (2005). Review of recent research into cellulosic whiskers, their properties and their application in nanocomposite field. Biomacromolecules 6: 612-626.

Baganz F, Hayes A, Marren D, Gardner DC, et al. (1997). Suitability of replacement markers for functional analysis studies in Saccharomyces cerevisiae. Yeast 13: 1563-1573.

Bakhrat A, Jurica MS, Stoddard BL and Raveh D (2004). Homology modeling and mutational analysis of Ho endonuclease of yeast. Genetics 166: 721-728.

Bradford MM (1976). Rapid and sensitive method for the quantitation of microgram quantities of protein utilizing the principle of protein-dye binding. Anal. Biochem. 72: 248-254.

Chen L, Drake MR, Resch MG, Greene ER, et al. (2014). Specificity of O-glycosylation in enhancing the stability and cellulose binding affinity of Family 1 carbohydrate binding modules. Proc. Natl. Acad. Sci. U. S. A. 111: 7612-7617.

De Vries RP (2003). Regulation of Aspergillus genes encoding plant cell wall polysaccharide-degrading enzymes; relevance for industrial production. Appl. Microbiol. Biotechnol. 61: 10-20.

Gao L, Gao F, Wang L, Geng C, et al. (2012). N-glycoform diversity of cellobiohydrolase I from Penicillium decumbens and synergism of nonhydrolytic glycoform in cellulose degradation. J. Biol. Chem. 287: 15906-15915.

Ghosh SK, Hajra S and Jayaram M (2007). Faithful segregation of the multicopy yeast plasmid through cohesin-mediated recognition of sisters. Proc. Natl. Acad. Sci. U. S. A. 104: 13034-13039.

Gietz RD and Woods RA (2002). Transformation of yeasts by the lithium acetate/single-stranded carrier/polyethylene glycol method. Methods Enzymol. 350: 87-96.

Harju S, Fedosyuk H and Peterson KR (2004). Rapid isolation of yeast genomic DNA: Bust n' Grab. BMC Biotechnol. 
4: 8.

Hector RE, Dien BS, Cotta MA and Qureshi N (2011). Engineering industrial Saccharomyces cerevisiae strains for xylose fermentation and comparison for switchgrass conversion. J. Ind. Microbiol. Biotechnol. 38: 1193-1202.

Hong J, Tamaki H, Akiba S, Yamamoto K, et al. (2001). Cloning of a gene encoding a highly stable endo-1,4- $\beta$-glucanase from Aspergillus niger and its expression in yeast. J. Biosci. Bioeng. 92: 434-441.

Horn SJ, Vaaje-Kolstad G, Westereng B and Eijsink VG (2012). Novel enzymes for the degradation of cellulose. Biotechnol. Befuels 5: 45.

Jeon E, Hyeon JE, Suh DJ, Suh YW, et al. (2009). Production of cellulosic ethanol in Saccharomyces cerevisiae heterologous expressing Clostridium thermocellum endoglucanase and Saccharomycopsis fibuligera beta-glucosidase genes. Mol. Cells 28: 369-373.

Laemmli UK (1970). Cleavage of structural proteins during the assembly of the head of bacteriophage T4. Nature 227: 680-685.

Li CH, Wang HR and Yan TR (2012). Cloning, Purification, and Characterization of a Heat- and Alkaline-Stable Endoglucanase B from Aspergillus niger BCRC31494. Molecules 17: 9774-9789.

Liu L, Liu C, Zou S, Yang H, et al. (2013). Expression of cellulase genes in Saccharomyces cerevisiae via $\delta$-integration subject to auxotrophic markers. Biotechnol. Lett. 35: 1303-1307.

Maley F, Trimble RB, Tarentino AL and Plummer TH Jr (1989). Characterization of glycoproteins and their associated oligosaccharides through the use of endoglycosidases. Anal. Biochem. 180: 195-204.

Miller GL (1959). Use of dinitrosalicylic acid reagent for determination of reducing sugar. Anal. Chem. 31: 426-428.

Teather RM and Wood PJ (1982). Use of Congo red-polysaccharide interactions in enumeration and characterization of cellulolytic bacteria from the bovine rumen. Appl. Environ. Microbiol. 43: 777-780.

Turon X, Rojas OJ and Deinhammer RS (2008). Enzymatic kinetics of cellulose hydrolysis: a QCM-D study. Langmuir. 24: 3880-3887.

Voth WP, Richards JD, Shaw JM and Stillman DJ (2001). Yeast vectors for integration at the HO locus. Nucleic Acids Res. 29: E59.

Walker ME, Gardner JM, Vystavelova A, McBryde C, et al. (2003). Application of the reuseable, KanMX selectable marker to industrial yeast: construction and evaluation of heterothallic wine strains of Saccharomyces cerevisiae, possessing minimal foreign DNA sequences. FEMS Yeast Res. 4: 339-347.

Yamada R, Tanaka T, Ogino C and Kondo A (2010a). Gene copy number and polyploidy on products formation in yeast. Appl. Microbiol. Biotechnol. 88: 849-857.

Yamada R, Taniguchi N, Tanaka T, Ogino C, et al. (2010b). Cocktail delta-integration: a novel method to construct cellulolytic enzyme expression ratio-optimized yeast strains. Microb. Cell Fact. 9: 32.

Yamada R, Hasunuma T and Kondo A (2013). Endowing non-cellulolytic microorganisms with cellulolytic activity aiming for consolidated bioprocessing. Biotechnol. Adv. 31: 754-763.

Zhang Y, Yin H and Lu H (2012). Recent progress in quantitative glycoproteomics. Glycoconj. J. 29: 249-258.

Genetics and Molecular Research 14 (2): 6439-6452 (2015) 